\title{
Perfil de crianças com transtorno do espectro autista
}

\author{
Profile of children with autism spectrum disorder \\ Perfil de los ninõs con trastorno del espectro autista
}

\section{Resumo}

Introdução: O Transtorno do Espectro Autista, também conhecido como autismo, é caracterizado como uma deficiência comunicativa, com comportamentos repetitivos e áreas restritas de interesse. Para isso, a identificação do perfil de crianças com a síndrome é de suma importância, o qual pode fornecer dados para efetivar políticas públicas. Objetivo: Este estudo tem como objetivo identificar o perfil de crianças com Transtorno do Espectro Autista. Método: Trata-se de uma pesquisa descritiva e exploratória com abordagem quantitativa, realizada em uma Associação de Amigos dos Autistas, na cidade de Teresina, Piauí, Brasil. Incluíram-se no estudo prontuários de crianças que estavam na faixa etária de zero a onze anos de idade atendidas em 2019. Resultados: Prevaleceu o sexo masculino (80,95\%), com idade entre 8 a 9 anos (44,4\%), Ensino fundamental ( $1^{\circ}$ a $4^{\circ}$ serie) (52,4\%). 61 (96,82\%) possuíam classificação para autismo infantil e utilizavam o tratamento medicamentoso com Risperidona (23/36,5\%). A maioria $(61 / 96,8 \%)$ realizava terapia individual e apresentava problemas associados à utilização do brinquedo, psicomotricidade, linguagem, socialização e limites. Conclusão: O estudo contribui para que profissionais sejam capazes de perceber as demandas dos autistas e colaborar com o seu melhor atendimento.

Palavras-chave: Transtorno do espectro autista; Transtorno autístico; Terapia da linguagem.

\begin{abstract}
Introduction: Autism Spectrum Disorder, also known as autism, is characterized as a communicative disability, with repetitive behaviors and restricted areas of interest. For this, the identification of the profile of children with the syndrome is of paramount importance, which can provide data to effect public policies. Objective: This study aims to identify the profile of children with Autism Spectrum Disorder. Method: This is a descriptive and exploratory research with a quantitative approach, carried out in an Association of Friends of the Autistic, in the city of Teresina, Piauí, Brazil. The study included medical records of children in the age group from zero to eleven years old attended in 2019. Results: The male gender (80.95\%), aged between 8 to 9 years (44.4\%) prevailed, Elementary school (1st to 4th grade) (52.4\%). 61 (96.82\%) had a classification for infantile autism and used drug treatment with Risperidone (23 / $36.5 \%)$. The majority $(61 / 96.8 \%)$ underwent individual therapy and had problems associated with the use of toys,
\end{abstract}


psychomotricity, language, socialization and limits. Conclusion: The study contributes so that professionals are able to perceive the demands of autistic people and collaborate with their best care.

Keywords: Autism spectrum disorder; Autistic disorder; Language therapy.

\section{Resumen}

Introducción: El trastorno del espectro autista, también conocido como autismo, se caracteriza por ser una discapacidad comunicativa, con conductas repetitivas y áreas de interés restringidas. Para ello es de suma importancia la identificación del perfil de los niños con el síndrome, que puede aportar datos para efectuar políticas públicas. Objetivo: Este estudio tiene como objetivo identificar el perfil de los niños con trastorno del espectro autista. Método: Se trata de una investigación descriptiva y exploratoria con enfoque cuantitativo, realizada en una Asociación de Amigos de los Autistas, en la ciudad de Teresina, Piauí, Brasil. El estudio incluyó historias clínicas de niños en el grupo de edad de cero a once años atendidos en 2019. Resultados: Predominó el género masculino (80,95\%), de 8 a 9 años $(44,4 \%)$, Educación primaria $\left(1^{\circ}\right.$ a $4^{\circ}$ grado) (52,4\%). 61 (96,82\%) tenían una clasificación para el autismo infantil y usaban tratamiento farmacológico con risperidona (23/36,5\%). La mayoría (61 / 96,8\%) se sometió a terapia individual y tuvo problemas asociados con el uso de juguetes, psicomotricidad, lenguaje, socialización y límites. Conclusión: El estudio contribuye para que los profesionales sean capaces de percibir las demandas de las personas autistas y colaboren con su mejor atención.

Palabras clave: Trastorno del espectro autista; Trastorno autístico; Terapia del lenguage.

\section{Introdução}

O transtorno do espectro autista (TEA) incide sobre aproximadamente 1\% da população geral (American Psychiatric Association, 2014). O TEA infantil consiste em problemática de saúde pública, tendo em vista ausência de consenso a respeito de sua etiologia, entretanto a tendência atual é considerá-la como síndrome de etiologia multicausal, envolvendo fatores genéticos, neurológicos e sociais da criança. O diagnóstico precoce é fundamental para estabelecer um tratamento adequado da criança, tendo em vista as necessidades singulares dessas crianças (Nascimento et al., 2016).

O TEA se manifesta de forma peculiar, caracterizado por alterações qualitativas nas interações sociais, na comunicação e no comportamento das crianças (Franzoi, Santos, Backes, \& Ramos, 2016). A criança com TEA apresenta uma tríade singular, a qual se caracteriza pela dificuldade e prejuízos qualitativos da comunicação verbal e não verbal, na interatividade social e na restrição do seu ciclo de atividades e interesses. Neste tipo de transtorno, podem também fazer parte da sintomatologia agitação intensa, frequência de estereotipias motoras à frustração, em episódios esporádicos e interesse incomum por aspectos sensoriais do ambiente, bem como presença de reações adversas a sons (Endres, 2015).

Deste modo, pensando nas características da criança com TEA e sabendo-se que seu desenvolvimento pode ser acometido de maneira global, é importante que esta criança seja vista em sua totalidade, abordando a esfera psicomotora, considerando primordialmente a maneira como ela realiza suas ocupações, visando qualidade de vida e independência, amenizando a complexidade e desafios vivenciados pela família e profissionais de saúde responsáveis por essa missão (Santos, 2017). Para isso, a identificação do perfil de crianças com a síndrome é de suma importância, o qual pode fornecer dados para efetivar políticas públicas. Assim, o objetivo do estudo foi identificar o perfil de crianças com Transtorno do Espectro Autista.

\section{Metodologia}

Trata-se de estudo descritivo e exploratório, com abordagem quantitativa. Segundo Koche (2011), na pesquisa exploratória não se trabalha com a relação entre variáveis, mas com o levantamento da presença das variáveis e da sua caracterização quantitativa ou qualitativa. Nesses casos é necessário desencadear um processo de investigação que identifique a natureza do fenômeno e aponte as características essenciais das variáveis que se quer estudar.

A pesquisa foi desenvolvida em uma Associação de Amigos dos Autistas - AMA situada na zona norte da cidade de Teresina, Estado do Piauí. A associação foi fundada em 29 de janeiro de 2000 e atualmente, atende em média 130 indivíduos com Transtorno do Espectro Autista. Essa instituição tem por objetivo ajudar os pais e amigos de crianças autistas a 
encontrarem apoio e suporte técnico para educação e tratamento de seus filhos. Atualmente, a AMA oferece os seguintes profissionais: assistentes sociais, professores, educadores físicos, psicólogos, psicopedagogos, fisioterapeutas e fonoaudiólogos, dentre mais de 50 profissionais e diversas estratégias para melhor atender as crianças, assim criando vínculos, confiança e despertando o interesse da sociedade em geral para sensibilizar a respeito da causa e do tratamento.

As 63 crianças cadastradas na AMA representam a amostra (N) de crianças atendidas na AMA, a margem de erro (E) com nível de confiança de $95 \%(\mathrm{Z}=1,96)$ e variância máxima $(\mathrm{P}=0,50)$ terão estimativa do parâmetro do estudo - perfil da criança será de:

$$
\begin{aligned}
& \frac{E=z^{2} \cdot \sqrt{p} \cdot(1-P)}{N} \\
& E=1,96 \sqrt{\frac{0,5 \times 0,50}{61}} \\
& E=1,96 \sqrt{0,0040} \\
& E=1,96 \times 0,0632 \\
& E=12,90 \%
\end{aligned}
$$

Para definição do processo de amostra foram considerados os seguintes critérios de inclusão: prontuários de crianças na faixa etária de 0 a 11 anos que tinham diagnóstico de Transtorno do Espectro Autista e estavam cadastrados na AMA de Teresina-PI. Foram excluídas as crianças que não tinham laudo médico anexado no prontuário que comprovasse que essa criança tivesse a síndrome comportamental, bem como os prontuários que estivessem com informações incompletas.

A coleta de dados foi realizada por meio do preenchimento de um formulário, que buscou dados relacionados à caracterização das crianças autistas quanto as variáveis: idade, sexo, município de residência, com quem reside, atendimento realizado na AMA, atendimentos realizados em outros serviços, problemas associados ao autismo, medicação que a criança faz uso.

Os levantamentos dos dados foram coletados dos prontuários de crianças atendidas durante o ano de 2019. Os dados foram transcritos dos prontuários e registradas numa ficha planejada para ser registrada em uma planilha do Microsoft Excel.

A coleta dos dados foi realizada pelos pesquisadores do estudo. Os dados da planilha foram importados para o programa SPSS IBM versão 20. A análise estatística foi descritiva, pela leitura das frequências absolutas (n) e relativas (\%) já que o estudo abordou apenas variáveis qualitativas. Os resultados foram apresentados na forma de tabelas.

\section{Resultados}

A amostra foi composta por 63 crianças de ambos os sexos. A Tabela 1 apresenta o perfil sociodemográfico de crianças autistas. A maioria das crianças é do sexo masculino, com residência na cidade de Teresina, com idades entre 8 a 9 anos, residem com os pais, com escolaridade de primeira a quarta série do ensino fundamental e com frequência na AMA de 3 a 4 anos. 
Tabela 1. Caracterização sociodemográfica de crianças autistas da AMA. Teresina (PI), 2019.

\begin{tabular}{|c|c|c|c|}
\hline Fator de interesse & Variável & $\mathrm{N}$ & $\%$ \\
\hline \multirow[t]{2}{*}{ Sexo } & Feminino & 12 & 19,04 \\
\hline & Masculino & 51 & 80,95 \\
\hline \multirow[t]{3}{*}{ Cidade/Estado } & Teresina, Piauí & 61 & 96,82 \\
\hline & Timon, Maranhão & 1 & 1,58 \\
\hline & Sem registro & 1 & 1,58 \\
\hline \multirow[t]{3}{*}{ Idade } & 6 a 7 & 15 & 23,80 \\
\hline & 8 a 9 & 28 & 44,44 \\
\hline & 10 a 11 & 20 & 31,74 \\
\hline \multirow[t]{14}{*}{ Com quem reside } & Mãe & 8 & 12,69 \\
\hline & Pais & 19 & 30,16 \\
\hline & Mãe e irmão & 2 & 3,17 \\
\hline & Pai e irmão & 4 & 6,34 \\
\hline & Mãe, pai e irmão & 13 & 20,63 \\
\hline & Mãe, pai e irmã & 9 & 14,28 \\
\hline & Mãe e padrasto & 1 & 1,58 \\
\hline & Mãe e avó & 2 & 3,17 \\
\hline & Mãe, avós e irmão & 1 & 1,58 \\
\hline & Mãe, avós e tia & 1 & 1,58 \\
\hline & Avó, tia e prima & 1 & 1,58 \\
\hline & Avós & 1 & 1,58 \\
\hline & Sem resposta & 1 & 1,58 \\
\hline & Total & 63 & 100 \\
\hline \multirow[t]{7}{*}{ Escolaridade } & Sem escolaridade & 0 & 0 \\
\hline & Maternal II & 1 & 1,58 \\
\hline & Ensino fundamental $\left(1^{\circ}\right.$ a $4^{\circ}$ serie $)$ & 33 & 52,38 \\
\hline & Ensino fundamental $\left(5^{\circ}\right.$ a $6^{\circ}$ serie $)$ & 5 & 7,93 \\
\hline & Sem registro & 24 & 38,09 \\
\hline & 7 a 8 & 3 & 4,76 \\
\hline & 5 a 6 & 20 & 31,74 \\
\hline \multirow{4}{*}{$\begin{array}{l}\text { Tempo que frequenta a } \\
\text { AMA }\end{array}$} & 3 a 4 & 29 & 46,03 \\
\hline & 1 a 2 & 10 & 15,87 \\
\hline & Sem registro & 1 & 1,58 \\
\hline & Total & 63 & 100 \\
\hline
\end{tabular}

Fonte: Autores (2019).

$\mathrm{Na}$ Tabela 2, todas as crianças participantes da pesquisa possuíam diagnóstico com classificação do CID-10, destas 61 (96,82\%) possuíam classificação para F84.0 (autismo infantil), 1 (1,58\%) possuía associado ao F84.0 o CID F91.3 (distúrbio 
desafiador e de oposição), seguindo 1 (1,58\%) aos F84.0, F91.3, F90 (distúrbios da atividade e da atenção) e F46.3 (hiperatividade).

Tabela 2. Diagnóstico de crianças autistas da AMA. Teresina (PI), 2019.

\begin{tabular}{llll}
\hline & & N & \\
\hline Diagnóstico (CID-10) & F84 & 61 & 96,82 \\
& F84 e F91.3 & 1 & 1,58 \\
& F84; F91.3; F90; F46.3 & 1 & 1,58 \\
Ano de diagnóstico & Total & 63 & 100 \\
& 2012 a 2013 & 21 & 33,33 \\
& 2014 a 2015 & 22 & 34,92 \\
& 2016 a 2017 & 10 & 15,87 \\
& 2018 & 2 & 3,17 \\
& Sem registro & 8 & 12,69 \\
\hline
\end{tabular}

Fonte: Autores (2019).

Na Tabela 3, os dados retratam o tratamento medicamentoso utilizado pelas crianças, estando a Risperidona entre os fármacos mais consumidos, com 36,51\% (n=23). 
Research, Society and Development, v. 10, n. 4, e3710413880, 2021

(CC BY 4.0) | ISSN 2525-3409 | DOI: http://dx.doi.org/10.33448/rsd-v10i4.13880

Tabela 3. Medicações de crianças autistas da AMA. Teresina (PI), 2019.

\begin{tabular}{|c|c|c|c|}
\hline Fator de interesse & Variável & $\mathrm{n}$ & $\%$ \\
\hline \multirow[t]{3}{*}{ Medicações de uso contínuo } & Sim & 42 & 66,66 \\
\hline & Não & 21 & 33,33 \\
\hline & Total & 63 & 100 \\
\hline \multirow[t]{18}{*}{ Nome das medicações } & Risperidona & 23 & 36,50 \\
\hline & Risperidona e Depakene & 2 & 3,17 \\
\hline & Risperidona e Neosine & 1 & 1,58 \\
\hline & Risperidona, Daforin, Carbamazepina & 1 & 1,58 \\
\hline & Risperidona e Ritalina & 2 & 1,58 \\
\hline & Risperidona e Quetiapina & 1 & 1,58 \\
\hline & Risperidona e Carbamazepina & 1 & 1,58 \\
\hline & Risperidona e Fenobarbital & 1 & 1,58 \\
\hline & Neoleptil & 1 & 1,58 \\
\hline & Haldol & 1 & 1,58 \\
\hline & Fenobarbital & 1 & 1,58 \\
\hline & Aristab & 1 & 1,58 \\
\hline & Ritalina e Levozine & 1 & 1,58 \\
\hline & Ritalina & 2 & 1,58 \\
\hline & Olazanpine & 1 & 1,58 \\
\hline & Digoxina e Furosemida & 1 & 1,58 \\
\hline & Não faz uso & 21 & 33,33 \\
\hline & Total & 63 & 100 \\
\hline
\end{tabular}

Fonte: Autores (2019).

A Tabela 4 demonstra que a maioria das crianças realiza terapia individual com diversos profissionais como: psicólogos, terapeutas ocupacionais, fonoaudiólogos, educador físico, entre outros. Sobre as aptidões de crianças portadoras do TEA destacou-se que $26(41,26 \%)$ fazem uso da arte, como o desenho, recorte e colagens. 
Research, Society and Development, v. 10, n. 4, e3710413880, 2021

(CC BY 4.0) | ISSN 2525-3409 | DOI: http://dx.doi.org/10.33448/rsd-v10i4.13880

Tabela 4. Terapia de crianças autistas da AMA. Teresina (PI), 2019.

\begin{tabular}{llll}
\hline Fator de interesse & Variável & $\mathrm{n}$ & \multicolumn{2}{c}{$\%$} \\
\hline Terapia realizada na & Terapia individual & & 01 \\
AMA & Terapia em grupo & 0 & 3,17 \\
& Sem registro & 2 & 100 \\
& Total & 63 & 11,11 \\
Aptidões & Música & 7 & 1,58 \\
& Trabalho manual & 1 & 0 \\
& Tecnologias (como computador e vídeo) & 0 & 41,26 \\
& Artes (como desenho, recorte e colagens) & 26 & 0 \\
& Não tem aptidão & 0 & 12,69 \\
& Música/tecnologia/artes & 8 & 20,63 \\
& Trabalho manual/tecnologia/artes & 13 & 1,58 \\
& Todos & 1 & 11,11 \\
& Sem registro & 7 & 100 \\
\hline
\end{tabular}

Fonte: Autores (2019).

A Tabela 5 traz uma abordagem sobre os problemas associados às crianças autistas quanto a sua relação a utilização do brinquedo, psicomotricidade, linguagem, socialização e limites. 
Tabela 5. Problemas associados às crianças autistas da AMA. Teresina (PI), 2019.

\begin{tabular}{lccccccc}
\hline & \multicolumn{2}{c}{ Sim } & \multicolumn{2}{c}{ Não } & \multicolumn{2}{c}{ NR } \\
& N & \% & N & \% & N & $\%$ \\
\hline 1. Psicomotricidade & & & & & & \\
Apresenta agitação psicomotora & 27 & 42,85 & 29 & 46,03 & 7 & 11,11 \\
Apresenta retardo psicomotor & 30 & 47,61 & 32 & 50,79 & 1 & 1,58 \\
Apresenta agressividade & 24 & 38,09 & 31 & 49,20 & 8 & 12,69 \\
2. Linguagem, socialização e limites & & & & & & \\
Ecolalia? & 35 & 55,55 & 23 & 36,50 & 5 & 7,93 \\
Expressa frases? & 30 & 47,61 & 22 & 34,92 & 11 & 17,46 \\
Compreende a comunicação com o outro? & 53 & 84,12 & 4 & 6,34 & 6 & 9,52 \\
Expressa necessidades? & 38 & 60,31 & 17 & 26,98 & 8 & 12,69 \\
Inicia diálogos? & 29 & 46,03 & 21 & 33,33 & 13 & 20,63 \\
Imita? & 40 & 63,49 & 13 & 20,63 & 10 & 15,87 \\
Olha em direção ao outro? & 33 & 52,38 & 16 & 25,39 & 14 & 22,22 \\
Estabelece contato visual? & 35 & 55,55 & 19 & 30,15 & 9 & 14,28 \\
Sorri para o outro? & 41 & 65,07 & 6 & 9,52 & 16 & 25,39 \\
Aproxima-se do outro? & 31 & 49,20 & 17 & 26,98 & 15 & 23,80 \\
Chama para brincar? & 17 & 26,98 & 31 & 49,20 & 15 & 23,80 \\
Beija/abraça & 28 & 44,44 & 22 & 34,92 & 13 & 20,63 \\
Interage com adultos, crianças & 38 & 60,31 & 18 & 28,57 & 7 & 7,93 \\
\hline
\end{tabular}

Fonte: Autores (2019).

\section{Discussão}

O perfil de crianças com transtorno do espectro autista encontrado neste estudo é semelhante ao de outros estudos nacionais. A maioria dessas crianças é do sexo masculino, sendo 80,95\%. Estudo evidenciou a distribuição por sexo, sendo possível afirmar que o TEA é quatro vezes mais comum nas crianças do sexo masculino, e embora existam muitos estudos, ainda não se consta ao certo esse domínio (Leite, Meirelles, \& Milhomem, 2015).

Estudo realizado com crianças e adolescentes com autismo no Centro de Atenção Psicossocial Infanto juvenil da região metropolitana do Rio de Janeiro evidenciou que a maior parte dos usuários autistas residiam com a família (86\%), e seu domicílio se situava na área de abrangência do CAPSi (82\%), o que ratifica os resultados encontrados no presente estudo, em que 96,82\% das crianças possuíam residência na cidade de Teresina, sugerindo tendência à territorialização do cuidado, e 30,16\% moram com os pais, evidenciando seu papel essencial no tratamento (Lima, Couto, Solis, Oliveira, \& Delgado, 2017).

É válido enfatizar que há um alto índice de crianças com diagnóstico do transtorno do espectro autista com idade entre 8 a 9 anos. No total, 52,38\% estão no ensino fundamental, ou seja, a maioria se enquadra no resultado esperado, embora apresentem dificuldades para realização de atividades diárias. Verificou-se que algumas das crianças com TEA do ensino fundamental não chegam sequer a concluir o período letivo, devido às dificuldades em conseguirem se relacionar com outras crianças ou mudanças de rotina diária (Bricout et al., 2019).

Frente a essa situação, surgem desafios para a família ao se deparar com o diagnóstico de TEA, que tende a buscar e coletar mais informações sobre o diagnóstico estabelecido. Entende-se que, quanto mais cedo a criança for diagnosticada e iniciar o tratamento, maiores serão as possibilidades de desenvolvimento, dentro de suas capacidades físicas e mentais (Aguiar 
\& Pondé, 2017). De acordo com os dados encontrados, foi identificado que a maioria das crianças frequentam a AMA de 3 a 4 anos, o que significa uma frequência na participação das atividades ofertadas ao tratamento e às terapias. Com isso, há uma tendência à progressão no desenvolvimento de habilidades, no desenvolvimento motor, físico e comportamental.

Além disso, os resultados encontrados evidenciam sobre diagnósticos de crianças que frequentam a associação. Em contrapartida ao DSM-5, cerca de $70 \%$ dos portadores do transtorno do espectro autista podem ter um transtorno mental comórbido e $40 \%$ podem ter dois ou mais transtornos mentais associados (American Psychiatric Association, 2014). Neste estudo, 1,58\% das crianças com TEA apresentavam outros transtornos mentais, como distúrbio desafiador, de oposição, distúrbio da atividade, da atenção e hiperatividade.

É notório observar os desafios que profissionais da saúde encontram no trajeto do seu ofício, relacionados à integralidade da atenção, voltada na promoção e prevenção da saúde. Em virtude ao transtorno do espectro autista, pode explanar que há uma grande dificuldade em torno do profissional na identificação, que está correlacionada aos sinais e sintomas que porventura é semelhante aos outros transtornos, desde modo é necessário novas estratégias de avaliação e diagnóstico.

O presente estudo mostrou que há uma eminente predominância de autistas que utilizam medicações de uso contínuo. Entre os fármacos mais utilizados está a risperidona, com 36,5\%. Tais dados vão ao encontro de resultados encontrados em outra pesquisa (Caetano \& Gurgel, 2018), em que 69,02\% dos participantes faziam uso de psicofármacos e 42,03\% utilizavam a risperidona como medicação de uso ininterrupto.

Após o diagnóstico, o atendimento às pessoas com TEA objetiva ampliar e auxiliar a independência e a qualidade de vida do indivíduo, por meio da utilização de antipsicóticos que favorecem o controle de diversos sintomas do autismo, dentre os quais encontra-se a risperidona, que possui indicação para o tratamento de irritabilidade associada ao transtorno autista (Brasil, 2014).

Ao corroborar com este estudo, os resultados apontam que as terapias são importantes para estimulação da área cerebral afetada, além de contribuir para melhorar a comunicação verbal e não verbal, romper com os padrões de isolamento, reduzir os comportamentos estereotipados, estimular a autoexpressão e a manifestação da subjetividade. Isto converge com a literatura quando afirma que a experiência do uso de terapias, como a música e utilização de tecnologia de enfermagem no cuidado às crianças autistas no CAPSi foi positiva, já que propiciou novos modos de fazer/brincar, de desenvolver habilidades e de se relacionar com os outros. Trata-se de uma intervenção de enfermagem que oportunizou interação, novos comportamentos e estimulação da linguagem (Franzoi et al., 2016).

Por conta disso, pode-se dizer que a terapia deve ter como premissa favorecer ao desenvolvimento da criança, focando sempre nas principais dificuldades apresentadas pelo diagnóstico, pois ressalta um auxílio no contexto educacional (Pinto et al, 2016). Constata-se que os profissionais que trabalham com crianças com TEA têm como propósito desenvolver um planejamento que contemple a necessidade de cada paciente (Rocha, Capobianco, Brito, \& Santos, 2018).

$\mathrm{O}$ estudo evidenciou os tratamentos ofertados às crianças autistas da associação, sendo estes medicamentosos, individuais e em grupo, bem como o atendimento multiprofissional. Todas essas atividades ajudam no desenvolvimento, na concentração e favorece o contexto de convívio educacional e social da criança com autismo. Verificou-se que a terapia individual foi a mais frequente e as aptidões com maior afinidade e facilidade das crianças foram demonstradas em atividades que envolvem artes de colagens, desenhos e atividades de trabalho manual.

O Ministério da Saúde, em 2014, criou um conjunto de orientações para identificar o diagnóstico precoce e tratamento, abrangendo o Projeto Terapêutico Singular (PTS). Essas diretrizes foram criadas para colaborar com os familiares e com as equipes multiprofissionais para a atenção à saúde da criança com TEA no contexto do Sistema Único de Saúde 
(SUS), tendo como base a avaliação das necessidades do indivíduo que serão abordadas no intuito de melhorar a qualidade, autonomia, independência e inserção social e familiar (Nascimento, Castro, Lima, Albuquerque, \& Bezerra, 2018).

A maneira de apresentar o projeto terapêutico singular possibilita aprofundar vínculos com a equipe e com a própria criança autista, configurando-se em prática enriquecedora em todos os seus contextos de vida. Desta maneira, o PTS tem a possibilidade de potencializar e estimular o cérebro, para que as crianças possam ter ganhos futuros e desenvolverem habilidades dialógicas e interação com o usuário (Cipriano \& Almeida, 2016).

É importante explicitar que mesmo utilizando o PTS as crianças autistas apresentam problemas associados ao transtorno. De acordo com o item referente à ecolalia, pôde-se perceber que mais da metade das crianças tinham ecolalias, um fenômeno persistente que se caracteriza como distúrbio de linguagem, definida como a repetição em eco da fala do outro, nesse sentido, é possível perceber que a caracterização da amostra é compatível a outro estudo de metodologia semelhante (Oliveira, Feldman, Couto, \& Lima, 2017).

Além do exposto, contatou-se que as crianças com TEA apresentam fragilidades que dificultam suas tarefas diárias, como birras, comportamentos heteroagressivos e autoagressividade, além de interesses específicos por determinados objetos ou temas, estereotipias e comportamentos não-funcionais e atípicos (Soto, Giserman Kiss, \& Carter, 2016).

Os resultados deste estudo assemelham-se a outro publicado ${ }^{(17)}$ onde afirma-se que pelo fato do diagnóstico do TEA comprometer o neurodesenvolvimento da criança, pode-se afirmar que esse transtorno provocará danos no desempenho do seu cérebro, gerando possivelmente prejuízos em sua evolução, como por exemplo, retardos na fala, no aprendizado e consequentemente na obtenção da sua função motora.

Ademais, percebe-se que a presença de estereotipias pode marcar uma forma de comunicação que permite interagir com outras pessoas e que visa estimular a autonomia para o desenvolvimento da linguagem (Endres, 2015). Os sintomas devem estar presentes desde a primeira infância e todos esses fatores resultam em uma inadequação pragmática que persiste até a vida adulta. Essas dificuldades em interpretar a ambiguidade lexical podem decorrer em parte, dos déficits no conhecimento semântico e no processamento da sentença. Além do que, podem também refletir comprometimentos das habilidades de memória que são necessárias para desenvolver representações mentais de estímulos verbais (Ishihara, Tamanaha, \& Perissinoto, 2016). Esse transtorno varia quanto à intensidade e à apresentação de traços ou sinais, sempre presente de forma precoce, geralmente antes dos três anos de idade, apresentando desvios qualitativos na comunicação, imaginação e na interação social que, em conjunto, caracterizam a tríade de dificuldades, que define a perda de contato interpessoal (Elder, Kreider, Brasher, \& Ansell, 2017).

As limitações deste estudo relacionam-se à falta de algumas informações nos prontuários das crianças, o que impede a generalização dos achados, no entanto, estes são considerados válidos, pois refletem condições semelhantes verificadas em pesquisas de maior abrangência, destacando-se a necessidade de estudos complementares que envolvam o tema, visto que ainda existem poucos estudos referentes a temática.

\section{Conclusão}

Este estudo evidenciou o perfil de crianças autistas atendidos na AMA, o que poderá contribuir para que os profissionais e a população em geral tenham ciência de algumas características de uma criança autista, já que o autismo pode se manifestar de forma muito peculiar entre diferentes crianças, e em uma mesma criança, de uma fase a outra do desenvolvimento.

Dessa forma, sabe-se que é de extrema importância que a equipe multidisciplinar esteja capacitada, principalmente os profissionais de Enfermagem, para poder identificar uma criança com TEA e conhecer o perfil de crianças autistas, o que poderá direcioná-los a terem uma visão holística, cabendo ao enfermeiro fazer uma escuta qualificada, de forma que saibam 
quais são as verdadeiras necessidades enfrentadas pelo seu paciente, sendo ele autista, e quais as intervenções necessárias a se fazer, promovendo uma qualidade de vida melhor tanto para as crianças e suas famílias.

Com isso, a intenção além de traçar o perfil das crianças é trazer essa devolutiva para os profissionais assistentes, pais e responsáveis, com o intuito de contribuir para o trabalho colaborativo, sugerindo-se assim a realização de trabalhos futuros que promovam intervenções nesse público para avaliar sua eficácia na independência das crianças autistas.

\section{Referências}

Aguiar, M. C. M. \& Pondé, M. P. (2017). Exclusion within the inclusion of children with autism spectrum disorder in the regular school system. Revista Debates em Psiquiatria, 3, 6-11. https://doi.org/10.25118/2236-918X-7-3-2

American Psychiatric Association. (2014). DSM-5: Manual diagnóstico e estatístico de transtornos mentais. Artmed Editora. http://www.niip.com.br/wpcontent/uploads/2018/06/Manual-Diagnosico-e-Estatistico-de-Transtornos-Mentais-DSM-5-1-pdf.pdf

Brasil. (2014). Diretrizes de Atenção à Reabilitação da Pessoa com Transtornos do Espectro do Autismo (TEA). https://bvsms.saude.gov.br/bvs/publicacoes/diretrizes_atencao_reabilitacao_pessoa_autismo.pdf

Bricout, V. A., Pace, M., Dumortier, L., Miganeh, S., Mahistre, Y., Guinot, M. (2019). Motor Capacities in Boys with High Functioning Autism: Which Evaluations to Choose? J Clin Med, 8(10), 1521. https://doi.org/10.3390/jcm810152

Caetano, M. V. \& Gurgel, D. C. (2018). Nutritional profile of children bearing autism spectrum disorder. Rev Bras Promoç Saúde, 31(1), 1-11. https://doi.org/10.5020/18061230.2018.6714

Cipriano, M. S. \& Almeida, M. T. P. (2016). O brincar como intervenção no transtorno do espectro do autismo. Revista Extensão em ação, 2(11), 78-91. https://doi.org/10.32356/exta.v2.n11.11832

Elder, J. H., Kreider, C. M., Brasher, S.N., Ansell, M. (2017). Clinical impact of early diagnosis of autism on the prognosis and parent-child relationships. Psychology Research and Behavior Management, 10, 283-292. https://doi.org/10.2147/PRBM.S117499

Endres, R. G. (2015). O Fenótipo Ampliado do Autismo em genitores de crianças com Transtorno do Espectro Autista - TEA. Psicologia: Teoria e Pesquisa, 31(3), 285-292. https://doi.org/10.1590/0102-37722015032268285292

Franzoi, M. A. H., Santos, J. L. G., Backes, V. M. S., Ramos, F. R. S. (2016). Intervenção musical como estratégia de cuidado de enfermagem a crianças com transtorno do espectro do autismo em um centro de atenção psicossocial. Texto \& Contexto-Enfermagem, 25(1). https://doi.org/10.1590/0104070720160001020015

Ishihara, M. K., Tamanaha, A. C., Perissinoto, J. (2016). Comprehension of ambiguity for children with Specific Language Impairment and Autism Spectrum Disorder. CoDAS, 28(6), 753-757. https://doi.org/10.1590/2317-1782/20162015260

Koche, J. C. (2011). Fundamentos de metodologia científica. Petrópolis: Vozes. Recuperado de: http://www.brunovivas.com/wpcontent/uploads/sites/10/2018/07/K\%C3\%B6che-Jos\%C3\%A9-Carlos0D0AFundamentos-de-metodologia-cient\%C3\%ADfica-_-teoriada0D0Aci\%C3\%AAncia-e-inicia\%C3\%A7\%C3\%A3o-\%C3\%A0-pesquisa.pdf.

Leite, R., Meirelles, L. M. A., Milhomem, D. B. (2015). Medicamentos usados no tratamento psicoterapêutico de crianças autistas em Teresina-PI. Boletim Informativo Geum, 6(3), 91-97. https://revistas.ufpi.br/index.php/geum/article/view/4377/3073

Lima, R. C., Couto, M. C. V., Solis, F. P., Oliveira, B. D. C., Delgado, P. G. G. (2017). Psychosocial Care for Children and Adolescents with Autism in the CAPSi of the Rio de Janeiro Metropolitan Area. Saúde e Sociedade, 26(1), 196-207. https://doi.org/10.1590/s0104-12902017168443

Nascimento, P. S., Zanon, R. B., Bosa, C. A., Nobre, J. P. S., Freitas Júnior, A. D. et al. (2016). Comportamentos de crianças do Espectro do Autismo com seus pares no contexto de educação musical. Revista Brasileira de Educação Especial, 21(1), 93-110. https://doi.org/10.1590/S1413-65382115000100007

Nascimento, Y. C. M. L., Castro, C. S. C., Lima, J. L. R., Albuquerque, M. C. S., Bezerra, D. G. (2018). Transtorno do espectro autista: detecção precoce pelo enfermeiro na estratégia saúde da família. Revista Baiana de Enfermagem, 32, e25425. https://doi.org/10.18471/rbe.v32.25425

Oliveira, B. D. C., Feldman, C., Couto, M. C. V., Lima, R. C. (2017). Políticas para o autismo no Brasil: entre a atenção psicossocial e a reabilitação. Revista Physis, 27(3), 707-726. https://doi.org/10.1590/s0103-73312017000300017

Pinto, R. N. M., Torquato, I. M. B., Collet, N., Reichert, A. P. S., Neto, V. L. S., Saraiva, A. M. (2016). Autismo infantil: impacto do diagnóstico e repercussões nas relações familiares. Revista Gaúcha de Enfermagem, 37(3), e61572. https://doi.org/10.1590/1983-1447.2016.03.61572

Rocha, A. N. D. C., Capobianco, N. A. N., Brito, L. B., Santos, A. R. (2018). Intervenção junto a indivíduos com transtorno do espectro do autismo: a percepção do profissional. Revista Educação Especial, 31(61), 417-30. https://doi.org/10.5902/1984686X23893

Santos, L. F. (2017). O transtorno do espectro autista e sua singularidade: uma proposta inclusiva calcada no ensino personalizado. Revista @ mbienteeducação, 10(1), 101-16. https://doi.org/10.26843/ae19828632v10n12017p85a100

Soares, A. M. \& Cavalcante Neto, J. L. (2015). Avaliação do comportamento motor em crianças com transtorno do espectro do autismo: uma revisão sistemática. Revista Brasileira de Educação Especial, 21(3), 445-458. https://doi.org/10.1590/S1413-65382115000300010 
Research, Society and Development, v. 10, n. 4, e3710413880, 2021

(CC BY 4.0) | ISSN 2525-3409 | DOI: http://dx.doi.org/10.33448/rsd-v10i4.13880

Soto, T., Giserman Kiss, I., Carter, A. S. (2016). Symptom presentations and classification of autism spectrum disorder in early childhood: application to the diagnostic classification of mental health and developmental disorders of infancy and early childhood. Infant Mental Health Journal, $37(5)$, 486-497. https://doi.org/10.1002/imhj.21589 